\title{
Stellungnahme der ZKBS zur Einstufung der Parasiten Leishmania brasiliensis, Leishmania donovani, Plasmodium falciparum und Trypanosoma brucei rhodesiense als Empfängerorganismen bei gentechnischen Arbeiten
}

entechnische Arbeiten mit den einzelligen Parasiten Leishmania brasiliensis, Leishmania donovani, Plasmodium falciparum und Trypanosoma brucei rhodesiense als Empfängerorganismen sind in der Sicherheitsstufe 3 durchzuführen, sofern mit Überträgern, in diesem Fall blutsaugenden Insekten, gearbeitet wird. Dabei kann von lüftungstechnischen Sicherheitsmaßnahmen der Stufe 3 abgesehen werden. Gentechnische Arbeiten ohne Überträger rechtfertigen die Einstufung in die Sicherheitsstufe 2, wenn Nukleinsäureabschnitte übertragen werden, die das Gefährdungspotenzial der o.g. Empfängerorganismen nicht erhöhen.

In der "Liste risikobewerteter Spender und Empfängerorganismen für gentechnische Arbeiten" sind die Parasiten L. brasiliensis, L. donovani, P. falciparum und T. brucei rhodesiense in die Risikogruppe $3^{* *}$ eingestuft. Diese Einstufung entspricht der in Anhang III der Arbeitnehmerschutzrichtlinie 2000/54/EG vom 18.9.2000 vorgenommenen Einstufung und bedeutet, dass bei bestimmten biologischen Arbeitsstoffen, die in die Risikogruppe 3 eingestuft und mit Sternchen $\left.{ }^{* *}\right)$ versehen wurden, das Infektionsrisiko für den Arbeitnehmer begrenzt ist, da eine Infektion über den Luftweg normalerweise nicht erfolgen kann. Daher kann auf bestimmte lüftungstechnische Sicherheitsmaßnahmen der Stufe 3 verzichtet werden. Da es nicht möglich war, unterschiedliche Stadien eines Parasiten getrennt in der Organismenliste aufzuführen, hat man die Möglichkeit der Markierung mit Indizes genutzt.

Der Index $\mathbf{f}^{1}$ gilt bei Arbeiten mit Überträgern oder infizierten Endwirten. Da Arbeiten mit einem Überträger besondere Risiken für den Experimentator bergen können, ist das Gefährdungspotenzial des infizierten Überträgers abzuwägen. Dem entsprechend sind gesonderte Sicherheitsvorkehrungen zu treffen, die sich an diesem Gefährdungspotenzial orientieren.

Der Index $\mathbf{d}^{2}$ bietet die Möglichkeit der Herabstufung der gentechnischen Arbeiten, sofern nicht mit Überträgern gearbeitet wird. Dies gilt auch für gentechnische Arbeiten mit den Parasiten L. brasiliensis, L. donovani, P. falciparum oder T. brucei rhodesiense als Empfängerorganismen: Wenn nicht vorgesehen ist, mit Überträgern zu arbeiten, können die Arbeiten in der Sicherheitsstufe 2 durchgeführt werden.

Auch in den Vorschriften zum Arbeitsschutz wie der TRBA 105 "Sicherheitsmaßnahmen bei Tätigkeiten mit biologischen Arbeitsstoffen der Risikogruppe $3^{* *}$ " werden unterschiedliche Stadien der o.g. Parasiten differenziert eingestuft und somit sind die infektiösen Stadien in Kultur oder im Säugetier der Risikogruppe 2 zugeordnet. Die Angleichung der gentechnikrechtlichen und arbeitnehmerschutzrechtlichen Regelungen erfolgt aus o.g. Gründen durch Heranziehung des Index d.

\footnotetext{
${ }^{1}$ Bei Arbeiten mit Überträgern oder infizierten Endwirten sind gesonderte Sicherheitsvorkehrungen zu treffen, die sich am Gefährdungspotenzial des Parasitenstadiums orientieren.

${ }^{2}$ Die Einstufung kann herabgesetzt werden, wenn die Arbeiten mit dem Parasiten ohne Überträger/Zwischenwirt durchgeführt werden.
} 